\title{
Low expression of DLC1 is predictive of poor therapeutic efficiency of fluoropyrimidine and oxaliplatin as adjuvant chemotherapy in gastric cancer
}

\author{
YUQI SU $^{1,2^{*}}$, LI LIN $^{1 *}$, JINGWEN ZHANG $^{1}$, YAQI JIANG ${ }^{1}$, CHANGQIE PAN $^{1}$, \\ LI SUN $^{1}$, JIANGMAN DUAN ${ }^{1}$ and WANGJUN LIAO ${ }^{1}$ \\ ${ }^{1}$ Department of Oncology, Nanfang Hospital, Southern Medical University, Guangzhou, Guangdong 510515; \\ ${ }^{2}$ Department of Oncology, The First People's Hospital of Yueyang, Yueyang, Hunan 414000, P.R. China
}

Received September 7, 2014; Accepted May 27, 2015

DOI: $10.3892 / \mathrm{mmr} .2015 .4173$

\begin{abstract}
The Rho-GTPase-activating protein, deleted in liver cancer-1 (DLC1), has been reported to be a tumor suppressor. However, the prognostic value of DLC1 in gastric cancer (GC) remains to be fully elucidated. Fluoropyrimidine-oxaliplatin (FP-LOHP) combination therapy has been widely used for the adjuvant chemotherapy of GC, however, no reliable marker has been identified to determine its efficiency. Thus, the present study performed a retrospective investigation involving 251 patients with stage IB-III GC, who received adjuvant chemotherapy following radical resection and 37 patients with stage IV GC, who underwent palliative resection. The expression of DLC1 was found to be reduced in the majority of GC samples (212/288 pairs of samples), compared with normal mucosa, in immunohistochemical analyses. Lower expression levels of DLC1 indicated a more advanced tumor-node-metastasis stage, increased lymph node metastasis, deeper tumor invasion, increased tumor size and a higher rate of distant metastasis. By contrast, relatively increased expression levels of DLC1 indicated a longer time to recurrence (TTR) [hazard ratio (HR), 2.232; $\mathrm{P}=0.004]$ and overall survival $(\mathrm{OS})$ rate (HR, 2.910; $\mathrm{P}=0.001)$. Patients receiving FP-LOHP adjuvant chemotherapy were significantly less likely to suffer GC recurrence $(\mathrm{P}=0.001)$ and succumb to mortality $(\mathrm{P}=0.004)$, compared with those who received alternative chemotherapies. However, only the patients with DLC1-positive GC receiving FP-LOHP [DLC1 (+)/FP-LOHP (+)] exhibited a more favorable TTR and OS, compared with the patients with DLC1 (+)/FP-LOHP (-)
\end{abstract}

Correspondence to: Professor Wangjun Liao, Department of Oncology, Nanfang Hospital, Southern Medical University, 1838 Guangzhou Avenue North, Guangzhou, Guangdong 510515, P.R. China

E-mail: nfyyliaowj@163.com

*Contributed equally

Key words: gastric cancer, deleted in liver cancer-1, fluoropyrimidine, oxaliplatin, prognosis
(TTR, $\mathrm{P}=0.001$; OS, $\mathrm{P}=0.020$ ). No significant improvement in clinical outcome was observed in GC patients with low DLC1 receiving FP-LOHP treatment (TTR, $\mathrm{P}=0.270$; OS, P=0.197). In conclusion, low expression of DLC1 correlated with GC progression and is predictive of higher rates of recurrence and mortality. Only patients with DLC1-positive GC may have an improved treatment outcome from the use of FP-LOHP as adjuvant chemotherapy.

\section{Introduction}

Gastric cancer (GC) is the fourth most common type of malignant tumor and the second leading cause of cancer-associated mortality worldwide (1). Although classic histopathological classifications have been used to predict the treatment outcomes and prognoses of GC, the survival and recurrence rates vary within individual histological classifications, which is possibly attributable to genetic heterogeneity. Extensive radical resection is the standard treatment for local stage GC, and adjuvant chemotherapy is recommended for patients with operable GC (2). However, the high recurrence rate remains the most common cause for treatment failure $(3,4)$. Thus, levels of sensitivity towards treatment differ significantly among patients with GC. At present, no reliable biomarkers have been identified for the assessment of the therapeutic effects of adjuvant chemotherapy in GC. In particular, no biomarkers predictive of the treatment outcome of fluoropyrimidine (fluorouracil or capecitabine) and oxaliplatin (FP-LOHP) combination therapy, widely used for GC adjuvant chemotherapy, are known.

Deleted in liver cancer-1 (DLC1) is a GTPase-activating protein, which exerts tumor suppressor activities $(5,6)$, and was first identified to be downregulated or deleted in $~ 50 \%$ of primary hepatocellular carcinomas (7). Consistently, the mRNA levels of DLC1 have been reported to be reduced in various types of cancer $(8,9)$, including lymphomas $(10)$, colorectal cancer (11) and gastric cancer (12). In previous studies, silencing of DLC1 has been demonstrated to promote cell proliferation $(13,14)$ and migration $(13,15)$, while restoration of the expression of DLC1 inhibits cell migration (16-18), proliferation and carcinogenesis (16). Although the protein expression status of DLC1 in GC, and its prognostic value, 
particularly in GC adjuvant chemotherapy, remain to be fully elucidated, DLC1 deficiency has been identified in GC cell lines (12). By contrast, low levels of DLC1 have been reported to indicate adverse prognosis in urothelial carcinoma (19), oral squamous cell carcinoma (20), lung cancer (21), cutaneous melanoma (22) and ovarian carcinoma (23). In addition, it has been previously reported that low expression levels of DLC1 are associated with chemoresistance of etoposide and carboplatin in lung cancer (24). This suggests that the expression status of DLC1 in GC may determine prognosis by affecting the responses to certain chemotherapeutic agents.

Taking these results into account, the present study hypothesized that the protein expression levels of DLC1 are downregulated in GC and these levels correlate with an adverse prognosis. The present study investigated whether the levels of DLC1 contributed to the treatment outcome of certain chemotherapeutic agents. Accordingly, immunohistochemical staining was used to examine the protein expression levels of DLC1 in GC and in the corresponding normal mucosa samples, and the relevance of DLC1 to the clinicopathological characteristics, survival rates, recurrence and clinical outcome of the adjuvant chemotherapy were analyzed.

\section{Materials and methods}

Patient follow-up and tissue samples. The present study was approved by the Ethics Review Board of Nanfang Hospital, Southern Medical University (Guangzhou, China). Samples for diagnostic purposes were obtained with the written informed consent of each patient. The tissue samples were obtained from patients during surgical resection between 2000 and 2011 and were fixed in formalin and embedded in paraffin at Nanfang Hopsital. A total of 288 pairs of GC samples and matched para-cancerous tissues were obtained from patients diagnosed with GC by histopathological examination in Nanfang Hospital between 2000 and 2011. The clinical stages of the tumors were defined according to the National Comprehensive Cancer Network Guidelines (http://www.nccn.org/; Version 1.2014, Gastric Cancer). Patient follow-up included time to recurrence (TTR) and/or overall survival (OS) rates. TTR was defined as the time from the date of surgical resection to the date of recurrence, detected by magnetic resonance imaging (Achieva 3.0T; Philips, Amsterdam, Holland) or computed tomography (Brilliance iCT; Philips, Amsterdam, Holland), while OS was calculated from the time of surgery to the time of GC-specific mortality. Mortality resulting from other causes was defined as a censored event. All of the data were obtained from hospital records or telephone follow-up. The patients (stage IB-III) received different therapeutic regimens: Epirubicin, cisplatin and fluorouracil $(n=23)$; fluorouracil $(n=61)$; capecitabine $(n=20)$; paclitaxel and cisplatin $(n=32)$; docetaxel and cisplatin $(n=11)$; oxaliplatin and fluorouracil $(n=63)$; oxaliplatin and capecitabine $(n=41)$.

Immunohistochemistry. The 4- $\mu$ m-thick tissue sections, cut using a microtome (RM2235; Leica Microsystems GmbH, Wetzlar, Germany) were deparaffinizated in dimethyl-benzene subsequent to incubation at $55^{\circ} \mathrm{C}$ for $2 \mathrm{~h}$, then were washed successively with $100 \%, 95 \%, 90 \%, 80 \%$ and $70 \%$ ethanol. Following three washes with phosphate-buffered saline (PBS), antigen retrieval was performed by boiling the sections in $0.01 \mathrm{M}$ sodium citrate-hydrochloric acid ( $\mathrm{pH} \mathrm{6;} \mathrm{Guangzhou}$ Tuo Ke Da Biological Technology, Co., Ltd. (Guangzhou, China) for $3 \mathrm{~min}$ in a high pressure cooker (W12PCH402E; Meidi, Co., Ltd., Wuhan, China). The sections were cooled for $15 \mathrm{~min}$ at $37^{\circ} \mathrm{C}$ and were washed again in PBS. Endogenous peroxidase was inactivated by incubating the samples in $3 \%$ hydrogen peroxide for $10 \mathrm{~min}$, prior to incubation of the samples at $4^{\circ} \mathrm{C}$ overnight with a primary rabbit polyclonal antibody against DLC1 (1:100; Abcam, Cambridge, MA, USA) in a humidified chamber. Sodium citrate hydrochloric acid (Guangzhou Tuo Ke Da Biological Technology, Co, Ltd.) was dissolved in $2,000 \mathrm{ml}$ distilled water (to $\mathrm{pH} 6.0$ ) during antigen retrieval Tissues were then incubated with the universal IgG antibody conjugated to horseradish peroxidase (Beijing Zhongshan Jinqiao Biotechnology Co., Ltd., Beijing, China) for $30 \mathrm{~min}$ at $37^{\circ} \mathrm{C}$, followed by washing again with PBS. Diaminobenzidine solution (5\%; Beijing Kang Wei Shi Ji Biological Technology, Co., Ltd., Beijing, China) was used for color development. Finally, the sections were rinsed in distilled water, counterstained with hematoxylin (Beijing Ding Guo Chang Sheng Biological Technology, Co., Ltd., Beijing, China), dehydrated with ethanol and were mounted with transparent cover slips. For the negative control samples, the primary antibody was replaced with PBS.

Evaluation of immunohistochemical observations. Two pathologists evaluated the immunoreactivity scores of DLC1 in a blinded-manner. The immunoreactivity scores were calculated by multiplying the scores of the extent and intensity of staining. Based on the average percentage of positively stained cells/100 tumor cells in 10 high-power fields (BX51TRF; Olympus Corporation, Toyko, Japan), the extent of staining was scored as follows: 0 (0-10\%), 1 (11-25\%), 2 (26-50\%), 3 $(51-75 \%)$ and $4(>75 \%)$. The intensity of staining was scored as follows: 0 (negative), 1 (pale yellow), 2 (yellow or deep yellow) and 3 (brown or dark brown). The samples were divided into two groups according to the total scores: DLC1-negative (score of 0-5) and DLC1-positive (score of 6-12).

Statistical analysis. SPSS software, version 19.0 (IBM SPSS, Armonk, NY, USA) was used to perform statistical analyses. A $\chi^{2}$ test was used to assess the associations between clinicopathologic parameters and the expression of DLC1. DLC1 staining score comparisons were analyzed using one-way analysis of variance or Student's t-test. The Kaplan-Meier method was performed to evaluate the survival curve, constructed using GraphPad Prism 5 software (GraphPad Software, Inc., La Jolla, CA, USA), and a log-rank test was used to estimate differences between the survival curves. Univariate and multivariate analyses, using Cox proportional hazard regression, were performed to investigate the risk factors of mortality and recurrence. All P-values were two-tailed and $\mathrm{P}<0.05$ was considered to indicate a statistically significant difference.

\section{Results}

Patient characteristics. A total of 288 patients were followed up for OS, which comprised 251 patients with stage IB-III 
Table I. Expression of DLC1 and clinicopathological parameters in 288 patients with stage IB-IV gastric cancer.

\begin{tabular}{|c|c|c|c|c|}
\hline \multirow[b]{2}{*}{ Parameter } & \multirow[b]{2}{*}{$\mathrm{n}(\%)$} & \multicolumn{2}{|c|}{ Expression of DLC1 (n) } & \multirow[b]{2}{*}{ P-value ${ }^{a}$} \\
\hline & & High & Low & \\
\hline Age (years) & & & & 0.814 \\
\hline$\leq 55$ & $147(51.04)$ & 35 & 112 & \\
\hline$>55$ & $141(48.96)$ & 35 & 106 & \\
\hline Gender & & & & 0.260 \\
\hline Male & $132(45.80)$ & 28 & 104 & \\
\hline Female & $156(54.20)$ & 42 & 114 & \\
\hline TNM stage & & & & $<0.001$ \\
\hline I & $52(18.10)$ & 26 & 26 & \\
\hline II & $95(33.00)$ & 23 & 72 & \\
\hline III & $104(36.10)$ & 17 & 87 & \\
\hline IV & $37(12.80)$ & 4 & 33 & \\
\hline Lymph node metastasis & & & & $<0.001$ \\
\hline No & $45(15.60)$ & 23 & 22 & \\
\hline N1 & $73(25.30)$ & 25 & 48 & \\
\hline $\mathrm{N} 2$ & $68(23.60)$ & 11 & 57 & \\
\hline N3 & $102(35.40)$ & 11 & 91 & \\
\hline Tumor invasion & & & & 0.004 \\
\hline $\mathrm{T} 1$ & $45(15.60)$ & 20 & 25 & \\
\hline $\mathrm{T} 2$ & $66(22.90)$ & 17 & 49 & \\
\hline $\mathrm{T} 3$ & $78(27.10)$ & 16 & 62 & \\
\hline $\mathrm{T} 4$ & $99(34.40)$ & 17 & 82 & \\
\hline Tumor size $(\mathrm{cm})$ & & & & $<0.001$ \\
\hline$\leq 3$ & $127(44.10)$ & 47 & 80 & \\
\hline$>3$ & $161(55.90)$ & 23 & 138 & \\
\hline Distant metastasis & & & & 0.040 \\
\hline M0 & $251(87.20)$ & 66 & 185 & \\
\hline M1 & $37(12.80)$ & 4 & 33 & \\
\hline Tumor differentiation & & & & 0.098 \\
\hline Well & $43(14.90)$ & 16 & 27 & \\
\hline Moderate & $104(36.10)$ & 22 & 82 & \\
\hline Poor & $141(49.00)$ & 32 & 109 & \\
\hline
\end{tabular}

High and low expression were determined as total immunoreactivity scores of $0-5$ and $6-12$, respectively. ${ }^{2} \chi^{2}$ test, $\mathrm{P}<0.05$ was considered statistically significant. DLC1, deleted in liver cancer-1; TNM, tumor-node-metastasis. N0, no regional lymph node metastasis; N1, metastasis in 1-2 regional lymph nodes; N2, metastasis in 3-6 regional lymph nodes; N3, metastasis in seven or more regional lymph nodes; T1, tumor invades lamina propria, muscularis mucosae or submucosa; T2, tumor invades muscularis propria; T3, tumor penetrates subserosal connective tissue without invasion of visceral peritoneum or adjacent structures; T4, tumor invades serosa (visceral peritoneum) or adjacent structures; M0, no distant metastasis; M1, distant metastasis.

GC and 37 patients with stage IV GC. The 251 patients with stage IB-III GC that underwent D2 gastrectomy and postoperative adjuvant chemotherapy were followed up for TTR. None of these patients received chemoradiation or preoperative chemotherapy. Among the patients who received adjuvant chemotherapy, 104 received FP-LOHP adjuvant chemotherapy, while the remaining 147 received regimens, which did not involve FP-LOHP combination (Fig. 1A). The ages of the patients ranged between 25 and 85 years (median, 52 years). The follow-up durations ranged between 0.5 and 79.0 months (median, 26.3 months). The clinicopathological characteristics of the patients are presented in Table I.

Expression levels of DLC1 lower in GC samples, compared with normal gastric mucosa. The protein expression status of DLC1 was detected using immunohistochemistry in all pairs of paraffin-embedded GC samples and matched para-cancerous gastric tissues. Representative staining examples of normal gastric tissues and DLC1-positive and -negative GC tissues are presented in Fig. 1. It was revealed that DLC1 was predominantly 
A

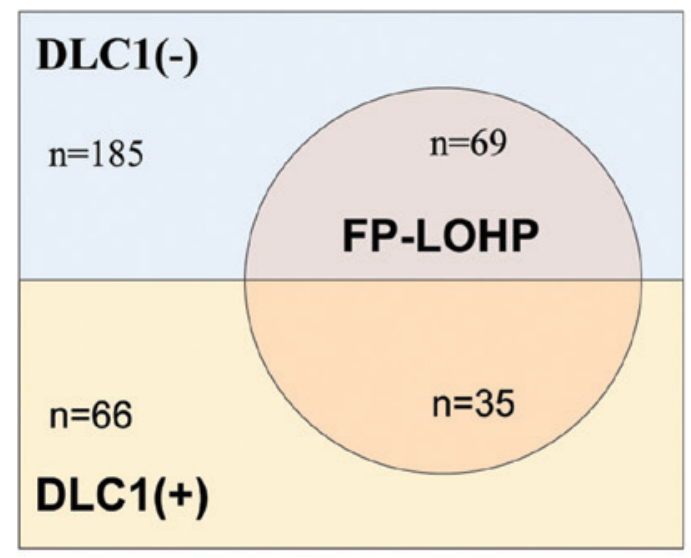

B

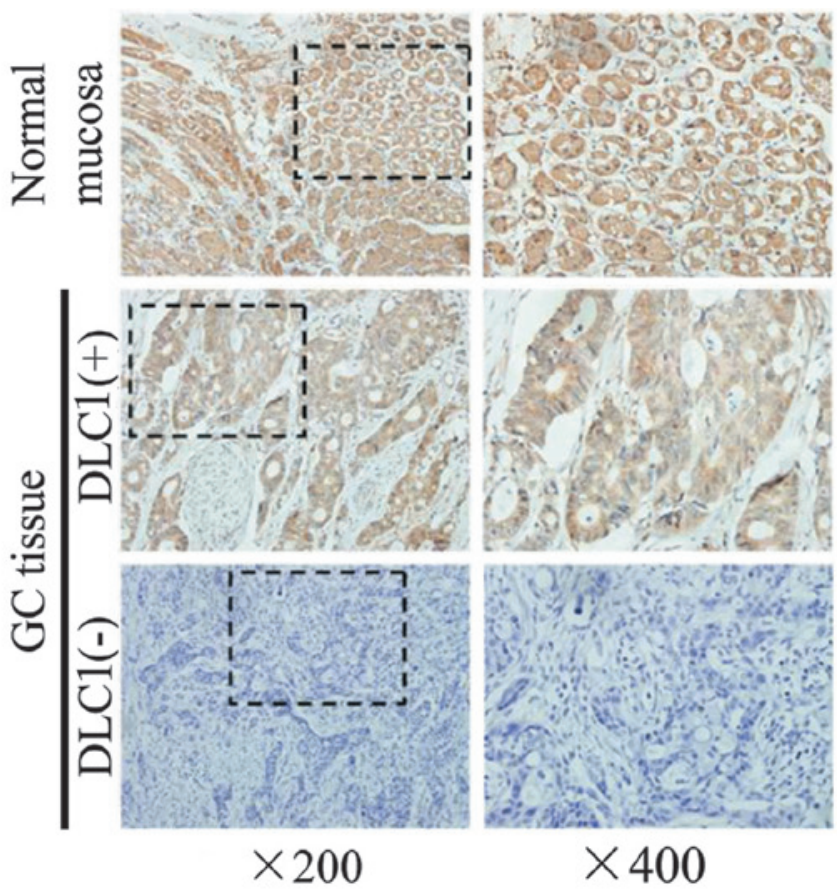

Figure 1. DLC1 expression status in GC. (A) A total of 251 stage IB-III patients underwent radical resection and adjuvant chemotherapy. Among these patients, 185 were defied as DLC1 (-) and 66 were DLC1 (+). A total of 69 DLC1-(-) and 35 DLC1-(+) patients received FP-LOHP [FP-LOHP (+)] as adjuvant chemotherapy, while the remaining patients received alternative therapies [FP-LOHP (-)]. (B) Representative images of DLC1 immunohistochemical staining in para-cancerous normal tissues and GC tissues, observed under an optical microscope. Dashed boxes indicate the areas visualized at magnification, $\mathrm{x} 400$ (on the right). DLC1, deleted in liver cancer-1; GC, gastric cancer; FP-LOHP, fluoropyrimidine-oxaliplatin combination therapy.

A

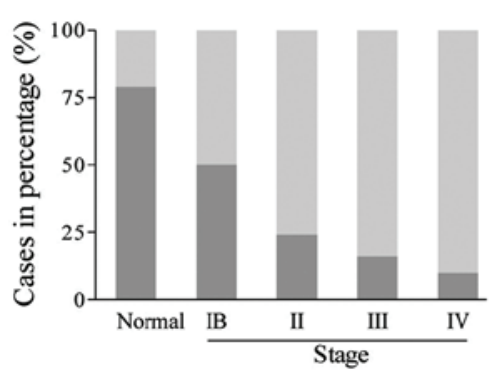

D

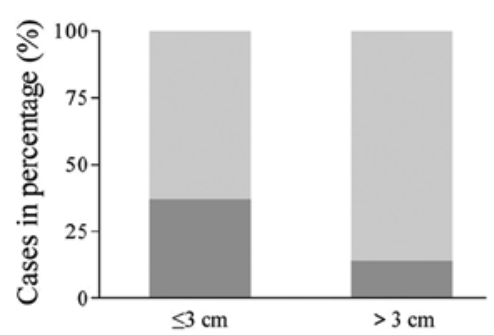

Tumor diameter

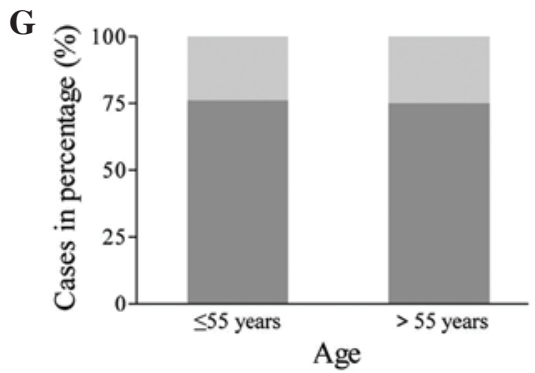

B

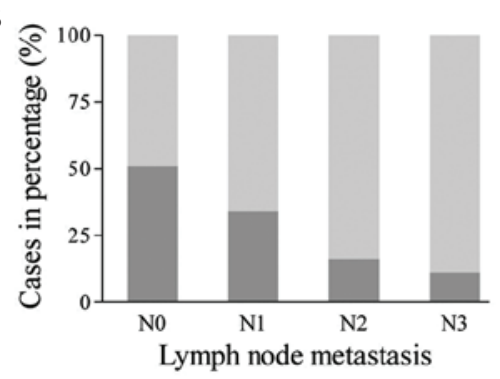

E

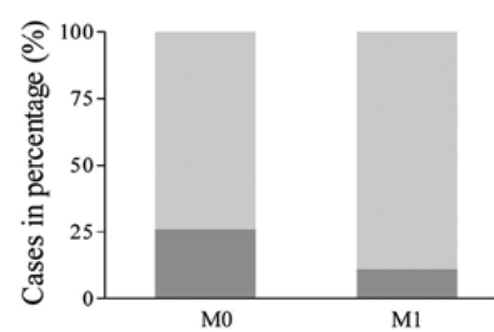

Distant metastasis

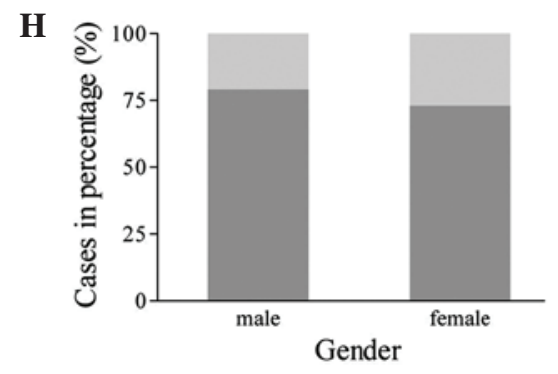

C

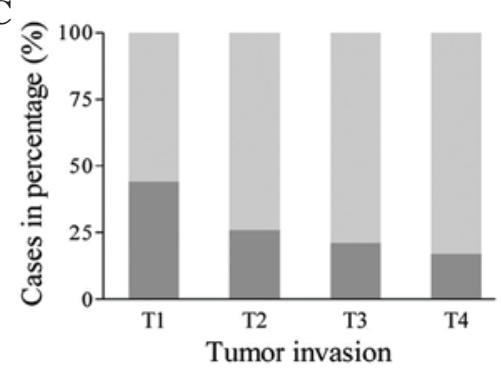

F

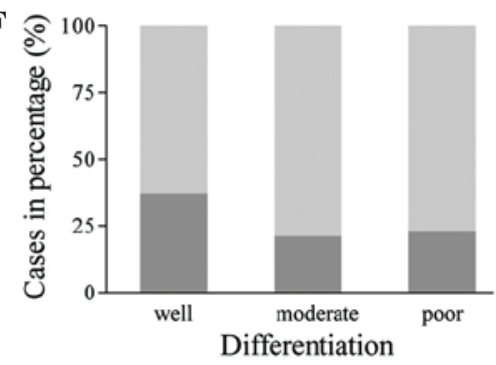

$\operatorname{DLC1}(-) \quad \square \operatorname{DLC} 1(+)$

Figure 2. DLC1 is frequently deficient in GC. (A) Negative expression of DLC1 was more frequently observed in GC samples, compared with the matched para-cancerous normal tissues $(\mathrm{P}<0.001)$, particularly in $\mathrm{GC}$ with increased $(\mathrm{A}) \mathrm{TNM}$ stage $(\mathrm{P}<0.001),(\mathrm{B})$ lymph node metastasis $(\mathrm{P}<0.001)$, $(\mathrm{C})$ invasion $(\mathrm{P}=0.004),(\mathrm{D})$ tumor diameter $(\mathrm{P}<0.001)$ and $(\mathrm{E})$ distant metastasis $(\mathrm{P}=0.040)$. The expression of DLC1 was not significantly associated with $(\mathrm{F})$ tumor differentiation $(\mathrm{P}=0.098),(\mathrm{G})$ age $(\mathrm{P}=0.814)$ or $(\mathrm{H})$ gender $(\mathrm{P}=0.260)$. DLC1, deleted in liver cancer-1; GC, gastric cancer; TNM, tumor-node-metastasis. 
Table II. Univariate analysis of the time to recurrence in patients with stage IB-III gastric cancer.

\begin{tabular}{lllr}
\hline Parameter & HR & 95\% CI & P-value \\
\hline Age $(>55$, vs. $\leq 55)$ & 1.029 & $0.739-1.431$ & 0.866 \\
Gender (female, vs. male) & 0.944 & $0.678-1.315$ & 0.733 \\
Tumor size (>3, vs. $\leq 3)$ & 6.681 & $4.528-9.857$ & $<0.001$ \\
Tumor differentiation (poor/moderate/well) & 4.663 & $3.326-6.538$ & $<0.001$ \\
TNM stage (IV/III/II/IB) & 5.979 & $4.302-8.309$ & $<0.001$ \\
Tumor invasion (T4/T3/T2/T1) & 2.599 & $2.148-3.146$ & $<0.001$ \\
Lymph node metastasis (N3/N2/N1/N0) & 2.785 & $2.296-3.378$ & $<0.001$ \\
Expression of DLC1 (low, vs. high) & 3.600 & $2.191-5.915$ & $<0.001$
\end{tabular}

HR, hazard ratio; CI, confidence interval; DLC1, deleted in liver cancer-1; TNM, tumor-node-metastasis; N0, no regional lymph node metastasis; N1, metastasis in 1-2 regional lymph nodes; N2, metastasis in 3-6 regional lymph nodes; N3, metastasis in seven or more regional lymph nodes; T1, tumor invades lamina propria, muscularis mucosae or submucosa; T2, tumor invades muscularis propria; T3, tumor penetrates subserosal connective tissue without invasion of visceral peritoneum or adjacent structures; $\mathrm{T} 4$, tumor invades serosa (visceral peritoneum) or adjacent structures; M0, no distant metastasis; M1, distant metastasis.

A

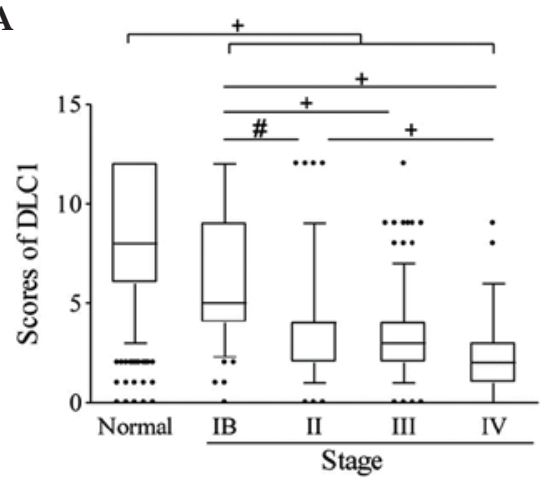

D

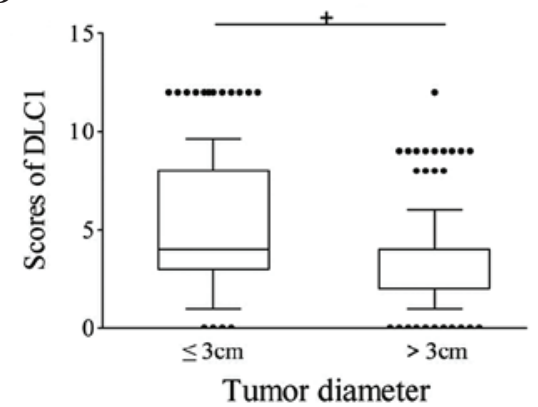

B

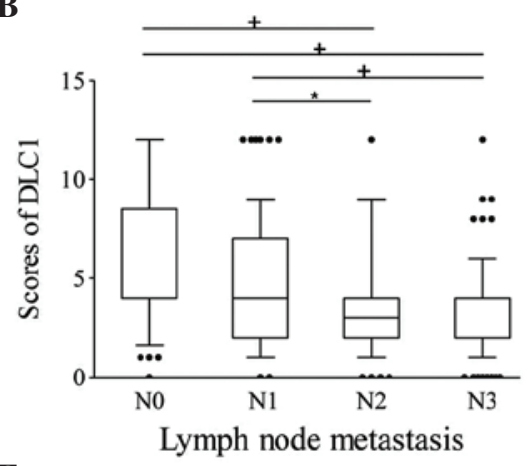

$\mathbf{E}$

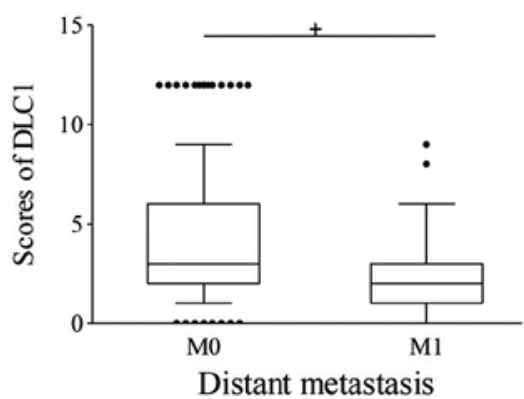

C

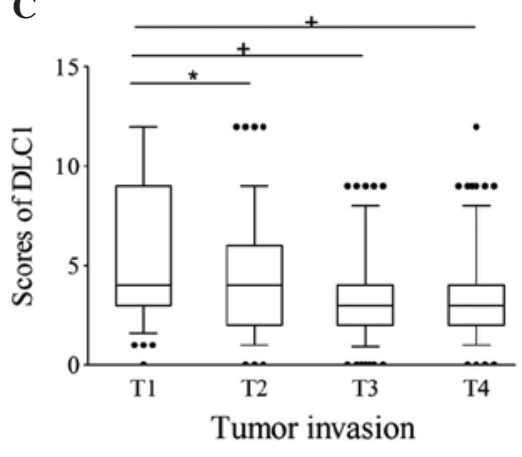

$\mathbf{F}$

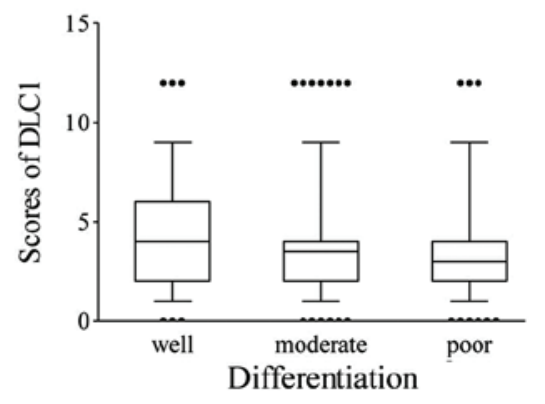

Figure 3. Expression of DLC1 is reduced in more advanced GC. Immunoreactivity scores of DLC1 were lower in GC samples with (A) later TNM stage, (B) increased lymph node metastasis, (C) increased tumor invasion, (D) larger tumor diameter and (E) increased distant metastasis. (F) No significant difference was observed among patients with GC with different degrees of differentiation. The lower and upper bars of the box plot represent the 10 and $90 \%$ percentile values. Error bars represent the $10 \%$ and $90 \%$ percentiles and dots below and above the box plots indicate values below or above the $10 \%$ and $90 \%$ percentiles, respectively. ${ }^{*} \mathrm{P}<0.05,{ }^{\text {}} \mathrm{P}<0.01,{ }^{+} \mathrm{P}<0.001$. GC, gastric cancer; DLC1, deleted in liver cancer- 1 ; TNM, tumor-node-metastasis.

expressed in the cytoplasm, and the immunoreactivity score of DLC1 was significantly lower in the majority of the pairs of GC samples, compared with the matched para-cancerous tissues [73.61\%; 212/288); $\mathrm{P}<0.001)$. As shown in Fig. 2A, DLC1 deficiency was more frequently observed in GC (75.69\%; 218/288), compared with the corresponding noncancerous gastric tissues (21.18\%; 61/288; $\mathrm{P}<0.001)$.

Expression of DLC1 decreases with the progression of $G C$. In order to elucidate the role of DLC1 in the progression of GC, the association between the levels of DLC1 and the clinicopathological characteristics of the patients was evaluated. Using a $\chi^{2}$ test, the expression level of DLC1 was be correlated with the tumor-node-metastasis (TNM) stage $(\mathrm{P}<0.001$; Fig. 2A), level of lymph node metastasis $(\mathrm{P}<0.001$; Fig. 2B), tumor invasion ( $\mathrm{P}=0.004$; Fig. 2C), tumor size $(\mathrm{P}<0.001$; Fig. 2D) and distant metastasis $(\mathrm{P}=0.04$; Fig. 2E). By contrast, no statistical significance was observed in the association between the expression of DLC1 and tumor differentiation status $(\mathrm{P}=0.098)$ (Fig. $2 \mathrm{~F})$, age $(\mathrm{P}=0.814$; 
Table III. Univariate analysis for mortality in patients with stage IB-IV gastric cancer.

\begin{tabular}{llrr}
\hline Parameter & HR & 95\% CI & P-value \\
\hline Age (>55, vs. $\leq 55)$ & 1.089 & $0.752-1.575$ & 0.653 \\
Gender (female, vs. male) & 0.910 & $0.629-1.317$ & 0.618 \\
Tumor size (>3, vs. $\leq 3)$ & 6.081 & $3.922-9.428$ & $<0.001$ \\
Tumor differentiation (poor/moderate/well) & 2.880 & $2.076-3.995$ & $<0.001$ \\
TNM stage (IV/III/II/IB) & 2.541 & $2.107-3.065$ & $<0.001$ \\
Tumor invasion (T4/T3/T2/T1) & 2.448 & $1.978-3.029$ & $<0.001$ \\
Lymph node metastasis (N3/N2/N1/N0) & 2.484 & $2.017-3.059$ & $<0.001$ \\
Expression of DLC1 (low, vs. high) & 4.056 & $2.264-7.266$ & $<0.001$
\end{tabular}

HR, hazard ratio; CI, confidence interval; DLC1, deleted in liver cancer-1; TNM, tumor-node-metastasis.

Table IV. Multivariate analysis for time to recurrence in patients with stage IB-III gastric cancer.

\begin{tabular}{lccc}
\hline Parameter & HR & 95\% CI & P-value \\
\hline Tumor size (>3, vs. $\leq 3)$ & 1.751 & $1.032-2.972$ & 0.038 \\
Tumor differentiation (poor/moderate/well) & 1.637 & $0.957-2.800$ & 0.072 \\
TNM stage (IV/III/II/IB) & 1.999 & $1.133-3.527$ & 0.017 \\
Tumor invasion (T4/T3/T2/T1) & 1.101 & $0.750-1.615$ & 0.623 \\
Lymph node metastasis (N3/N2/N1/N0) & 1.528 & $1.159-2.015$ & 0.003 \\
Expression of DLC1 (low, vs. high) & 2.232 & $1.295-3.857$ & 0.004 \\
\hline
\end{tabular}

HR, hazard ratio; CI, confidence interval; DLC1, deleted in liver cancer-1; TNM, tumor-node-metastasis.

Table V. Multivariate analysis for mortality in patients with stage IB-IV gastric cancer.

\begin{tabular}{|c|c|c|c|}
\hline Parameter & HR & $95 \% \mathrm{CI}$ & P-value \\
\hline Tumor size (>3, vs. $\leq 3)$ & 1.526 & $0.829-2.810$ & 0.175 \\
\hline Tumor differentiation (poor/moderate/well) & 1.318 & $0.933-1.862$ & 0.117 \\
\hline TNM stage (IV/III/II/IB) & 1.927 & $1.476-2.516$ & $<0.001$ \\
\hline Tumor invasion (T4/T3/T2/T1) & 1.550 & $1.178-2.039$ & 0.002 \\
\hline Lymph node metastasis (N3/N2/N1/N0) & 1.267 & $0.981-1.637$ & 0.070 \\
\hline Expression of DLC1 (low, vs. high) & 2.910 & $1.543-5.488$ & 0.001 \\
\hline
\end{tabular}

HR, hazard ratio; CI, confidence interval; DLC1, deleted in liver cancer-1; TNM, tumor-node-metastasis.

Fig. 2G) or gender ( $\mathrm{P}=0.260$; Fig. $2 \mathrm{H})$. In addition, the overall expression level of DLC1 was observed to be significantly reduced in the GC tissues, compared with the paired normal tissues (Fig. 3A). GC tissues of a more advanced TNM stage (Fig. 3A), with increased lymph node metastasis (Fig. 3B), infiltration (Fig. 3C), tumor sizes (Fig. 3D) and distant metastasis (Fig. 3E) exhibited lower expression levels of DLC1 expression levels. However, the tumor differentiation scores were not significantly different ( $\mathrm{P}=0.116$; Fig. $3 \mathrm{~F})$. The results obtained suggested that DLC1 tended to reduce during the progression of GC. The present study subsequently investigated the association between treatment outcome and the expression of DLC1.
Reduced expression of DLC1 indicates poor prognosis of GC. To investigate the prognostic value of the expression of DLC1 in GC, univariate and multivariate analyses were performed. Univariate analysis revealed that tumor size $(\mathrm{P}<0.001)$, tumor differentiation $(\mathrm{P}<0.001)$, TNM stage $(\mathrm{P}<0.001)$, tumor invasion $(\mathrm{P}<0.001)$, lymph node metastasis $(\mathrm{P}<0.001)$ and the expression of DLC1 $(\mathrm{P}<0.001)$ were correlated with GC recurrence (Table II). In addition, tumor size $(\mathrm{P}<0.001)$, tumor differentiation $(\mathrm{P}<0.001)$, TNM stage $(\mathrm{P}<0.001)$, tumor invasion $(\mathrm{P}<0.001)$, lymph node metastasis $(\mathrm{P}<0.001)$ and the expression of DLC1 $(\mathrm{P}<0.001)$ significantly affected the mortality rates of patients with GC (Table III). When these parameters were included in the multivariate analysis, the expression of 
Table VI. Efficiency of DLC1/FP-LOHP as an adjuvant chemotherapy for gastric cancer.

\begin{tabular}{|c|c|c|c|c|c|c|c|c|}
\hline \multirow[b]{2}{*}{ DLC1 } & \multirow[b]{2}{*}{ FP-LOHP } & \multirow[b]{2}{*}{$\mathrm{n}(\%)$} & \multicolumn{2}{|c|}{ TTR (months) } & \multirow[b]{2}{*}{ 3-year non-recurrence $(\%)$} & \multicolumn{2}{|c|}{ OS (months) } & \multirow[b]{2}{*}{5 -year survival $(\%)$} \\
\hline & & & Median & $95 \% \mathrm{CI}$ & & Median & $95 \% \mathrm{CI}$ & \\
\hline+ & + & $35(13.9)$ & l & / & 89.6 & / & l & 74.5 \\
\hline+ & - & $31(12.4)$ & 37 & $25.8-48.2$ & 51.4 & / & l & 58.6 \\
\hline- & + & $69(27.5)$ & 27 & $21.6-32.4$ & 32.9 & 51 & $33.2-68.8$ & 16.7 \\
\hline- & - & $116(46.2)$ & 22 & $14.3-29.7$ & 23.3 & 36 & $32.4-39.6$ & 28.2 \\
\hline
\end{tabular}

DLC1, deleted in liver cancer-1; FP-LOHP, combination of fluoropyrimidine plus oxaliplatin; TTR, time to recurrence; OS, overall survival time; CI, confidence interval.

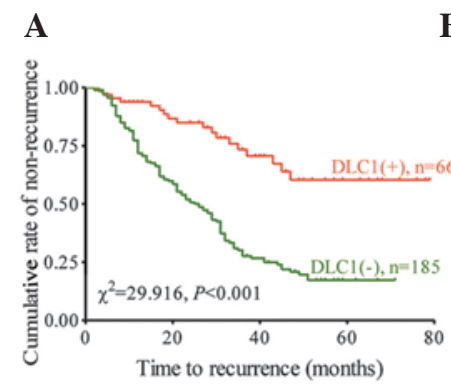

B

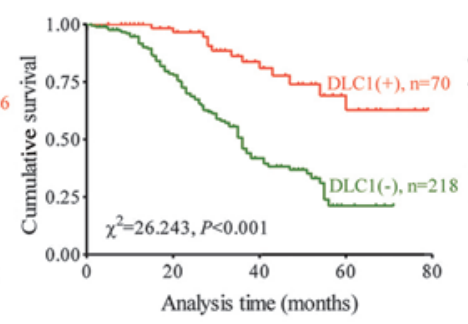

C



D

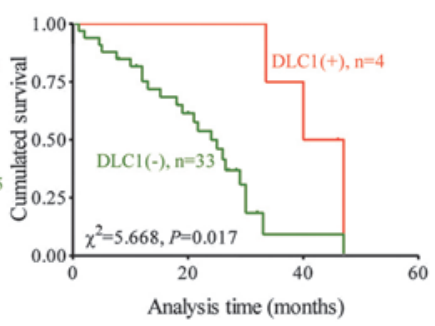

Figure 4. DLC1-negative patients are more likely to exhibit GC recurrence and shorter survival rates. Kaplan-Meier analysis was performed in (A) patients with stage IB-III GC for time to recurrence and in (B) patients with stage IB-IV GC for OS. Stratified analysis was also performed to evaluate the effect of DLC1 on OS in patients who (C) received radical resection (stage IB-III) and (D) underwent palliative surgery (stage IV). DLC1, deleted in liver cancer-1; GC, gastric cancer; OS, overall survival rate.

DLC1 remained a clear independent indicator of rates of recurrence $(\mathrm{P}=0.004$; Table IV) and mortality $(\mathrm{P}=0.001$; Table $\mathrm{V})$. The relative risks of GC recurrence [hazard ratio (HR), 2.232; 95\% confidence interval (CI), 1.295-3.847], presented in Table IV, and mortality (HR, 2.910; 95\% CI, 1.543-5.488], presented in Table V, in patients with DLC1-negative GC were 2-fold higher, compared with those in DLC1-positive patients. The above data suggested that the DLC1 deficiency, detected using immunohistochemistry, is predictive of a higher risk of recurrence and mortality in patients with GC.

To further examine the effect of negative expression of DLC1 on GC recurrence and mortality rates, TTR and OS were evaluated using Kaplan-Meier survival analysis. In the 251 patients with stage IB-III GC, TTR was significantly shorter in patients with DLC1-negative GC, compared with those with DLC1-positive GC (estimated 3-year recurrence-free survival rates, 30.1, vs. $73.4 \%$, respectively; Fig. 4A). In addition, in the 288 patients with stage IB-IV, those with DLC1-negative GC demonstrated significantly shorter OS rates, compared with those with DLC1-positive GC (estimated 5-year OS rate, 21.2 , vs. $62.8 \%$; Fig. 4B). Stratified analysis was also performed to evaluate the effect of DLC1 on OS in patients with stage IB-III GC, who received radical resection and those with stage IV GC, who underwent palliative surgery. DLC1 deficiency was indicative of reduced survival rates in patients with stage IB-III (Fig. 4C) and stage IV (Fig. 4D). Among the patients with DLC1-negative GC, the median TTR in those with stage IB-III GC was 26.0 months and the median OS in the patients with stage IB-IV GC was 36.0 months. However, more than half of the patients with DLC1-positive stage IB-III cancer remained relapse-free at the end of the follow-up.

High expression levels of DLCl indicate improved treatment outcome of FP-LOHP as an adjuvant chemotherapy. FP-LOHP is widely used as an adjuvant chemotherapy for GC. However, the sensitivity to FP-LOHP varies significantly among patients and, currently, there is no reliable biomarker available to predict the clinical response to this combination therapy. Therefore, the present study aimed to elucidate whether the expression levels of DLC1 affect the clinical outcome of FP-LOHP. In the present study, samples from 251 patients with stage IB-III GC were used. The median duration and rate of 3-year non-recurrence and 5-year survival rates for each group are presented in Table IV. Over half of the patients with DLC1-positive GC, who received FP-LOHP adjuvant chemotherapy [DLC1 (+)/FP-LOHP (+)] were recurrence-free at 3 years $(89.6 \%)$, with a 5-year-surival rate for DLC1-positive GC of $69.0 \%$, accordingly, the median durations of non-recurrence and survival was not determined (Table IV).

In the Kaplan-Meier analysis, patients receiving FP-LOHP as an adjuvant chemotherapy had lower rates of GC recurrence (Fig. 5A) and mortality (Fig. 5B), compared with those who underwent the alternative therapies. However, only the patients in the DLC1-positive group, who received FP-LOHP adjuvant chemotherapy [DLC1 (+)/FP-LOHP (+)] exhibited more favorable TTR (Fig. 5A) and OS (Fig. 5B) results, compared with those receiving the alternative chemotherapies 

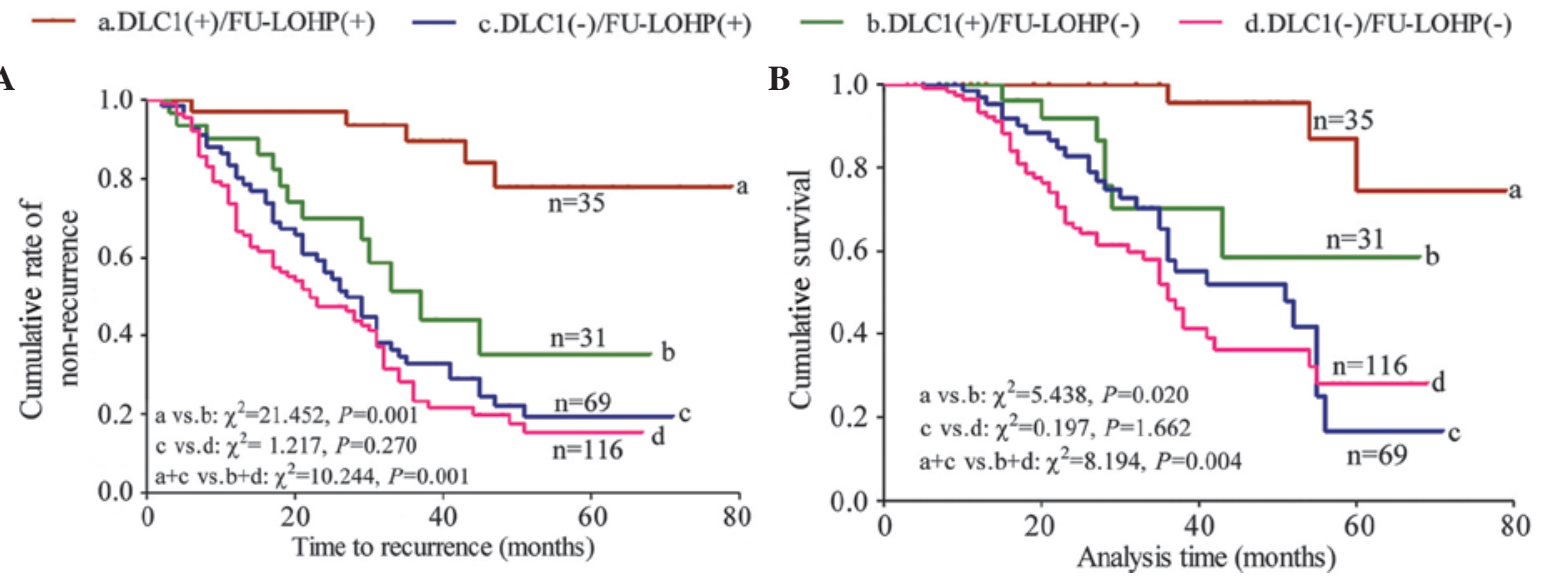

Figure 5. DLC1 levels determine the efficiency of FP-LOHP in GC adjuvant chemotherapy. (A) Recurrence-free curve and (B) survival curve of patients with stage IB-III GC. DLC1, deleted in liver cancer-1; FP-LOHP, fluoropyrimidine-oxaliplatin combination therapy; GC, gastric cancer.

[DLC1 (+)/FP-LOHP (-)]. The patients with DLC1-negative GC did not benefit from treatment with FP-LOHP (Fig. 5A for TTR and Fig. 5B for OS).

\section{Discussion}

In the present study, the expression levels of DLC1 in GC samples and adjacent normal mucosa samples were assessed using immunohistochemistry. To the best of our knowledge, the present study is the first to investigate the prognostic significance of the protein level of DLC1 in GC. DLC1 was identified to be significantly lower in the GC samples, compared with the normal gastric mucosa, particularly in tumors of later disease stages, with increased invasion, lymph node metastasis and distant metastasis. Patients with DLC1-negative GC exhibited shorter survival rates and higher risks of recurrence. By contrast, favorable outcomes of FP-LOHP adjuvant chemotherapy were observed only in the patients with DLC1-positive GC.

In previous studies, DLC1 has been reported to be a tumor suppressor, and its low level of expression indicates undesirable prognoses in numerous types of cancer (19-23). Loss of DLC1 has been reported to enhance cell growth $(13,14)$, migration $(13,15)$ and angiogenesis during tumorigenesis (25). Restoration of silenced DLC1 gene has been demonstrated to inhibit migration (16-18) and invasion $(17,18)$, induce apoptosis (16) in vitro and suppress progression and metastasis in vivo (17). However, at present, the prognostic value of DLC1 in GC remains to be fully elucidated. In the present study, DLC1 deficiency was more frequently observed in cases of GC with larger tumor size, more pronounced invasion, later TNM stage, and higher risks of local lymph node metastasis and distant metastasis. The above data indicated that DLC1 deficiency may be important in the process of tumor progression in GC. The present study also demonstrated that patients with DLC1-negative GC exhibited increased risks of tumor recurrence following radical resection and subsequent adjuvant chemotherapy. In addition, patients with DLC1-negative GC exhibited significantly shorter survival times, compared with those with DLC1-positive GC. These observations, consistent with previous studies in other malignances (19-23), indicated that negative expression of DLC1 predicts an undesirable prognosis in GC, which may be, in part, due to its potential involvement in the process of tumorigenesis and tumor progression.

The FP-LOHP combination has been widely applied in the treatment of GC, particularly in postoperative chemotherapy. The treatment inefficiency of this combination significantly increases the risk of postoperative recurrence in locally advanced GC. To the best of our knowledge, the present study is the first to indicate DLC1 as a novel biomarker, predictive of the treatment outcome of FP-LOHP adjuvant chemotherapy. The observations of the present study suggested that only patients with DLC1-positive expression benefit from FP-LOHP adjuvant chemotherapy. Thus, detection of the expression of DLC1 may be useful in predicting the treatment outcome of FP-LOHP adjuvant chemotherapy. However, this hypothesis requires validation by further investigations.

In conclusion, the present study demonstrated the prognostic value of DLC1. The expression of DLC1 reduced during the progression of $\mathrm{GC}$, indicating high risks of recurrence and mortality. The clinical outcome from FP-LOHP adjuvant chemotherapy was improved only in DLC1-positive patients with locally advanced GC. In conclusion, DLC1 may be a potential biomarker, indicating the therapeutic effect of FP-LOHP on the clinical outcome of GC.

\section{Acknowledgements}

The present study was supported by the National Natural Science Foundation of China (grant no. 31271564 to Professor Wangjun Liao), the Young Innovator Project granted by the Education Department of Guangdong Province (grant. no. 2014KQNCX043 to Dr. Li Lin) and the Special Foundation for National Clinical Specialties of China to the Department of Oncology, Nanfang Hospital. Part of the findings of the present study were presented as a poster presentation at the European Society for Medical Oncology Congress, Madrid, Spain, 26-30 September, 2014.

\section{References}

1. Siegel R, Naishadham D and Jemal A: Cancer statistics, 2013. CA Cancer J Clin 63: 11-30, 2013. 
2. Macdonald JS, Smalley SR, Benedetti J, Hundahl SA, Estes NC, Stemmermann GN, Haller DG, Ajani JA, Gunderson LL, Jessup JM, et al: Chemoradiotherapy after surgery compared with surgery alone for adenocarcinoma of the stomach or gastroesophageal junction. N Engl J Med 345 725-730, 2001.

3. Maehara Y, Hasuda S, Koga T, Tokunaga E, Kakeji Y and Sugimachi K: Postoperative outcome and sites of recurrence in patients following curative resection of gastric cancer. Br J Surg 87: 353-357, 2000.

4. Yoo CH, Noh SH, Shin DW, Choi SH and Min JS: Recurrence following curative resection for gastric carcinoma. Br J Surg 87: 236-242, 2000.

5. Wang Y, Lei R, Zhuang X, Zhang N, Pan H, Li G, Hu J, Pan X, Tao Q, Fu D, et al: DLC1-dependent parathyroid hormone-like hormone inhibition suppresses breast cancer bone metastasis. J Clin Invest 124: 1646-1659, 2014.

6. Barras D and Widmann C: GAP-independent functions of DLC1 in metastasis. Cancer Metastasis Rev 33: 87-100, 2014.

7. Yuan BZ, Miller MJ, Keck CL, Zimonjic DB, Thorgeirsson SS and Popescu NC: Cloning, characterization and chromosomal localization of a gene frequently deleted in human liver cancer (DLC-1) homologous to rat RhoGAP. Cancer Res 58: 2196-2199, 1998.

8. Low JS, Tao Q, Ng KM, Goh HK, Shu XS, Woo WL, Ambinder RF, Srivastava G, Shamay M, Chan AT, et al: A novel isoform of the $8 \mathrm{p} 22$ tumor suppressor gene DLC1 suppresses tumor growth and is frequently silenced in multiple common tumors. Oncogene 30: 1923-1935, 2011.

9. Seng TJ, Low JS, Li H, Cui Y, Goh HK, Wong ML, Srivastava G, Sidransky D, Califano J, Steenbergen RD, et al: The major 8p22 tumor suppressor DLC1 is frequently silenced by methylation in both endemic and sporadic nasopharyngeal, esophageal and cervical carcinomas and inhibits tumor cell colony formation. Oncogene 26: 934-944, 2007.

10. Ying J, Li H, Murray P, Gao Z, Chen YW, Wang Y, Lee KY, Chan AT, Ambinder RF, Srivastava G, et al: Tumor-specific methylation of the $8 \mathrm{p} 22$ tumor suppressor gene DLC1 is an epigenetic biomarker for Hodgkin, nasal NK/T-cell and other types of lymphomas. Epigenetics 2: 15-21, 2007.

11. Peng H, Long F, Wu Z, Chu Y, Li J, Kuai R, Zhang J, Kang Z, Zhang $X$ and Guan M: Downregulation of DLC-1 gene by promoter methylation during primary colorectal cancer progression. Biomed Res Int 2013: 181384, 2013.

12. Kim TY, Jong HS, Song SH, Dimtchev A, Jeong SJ, Lee JW, Kim TY, Kim NK, Jung M and Bang YJ: Transcriptional silencing of the DLC-1 tumor suppressor gene by epigenetic mechanism in gastric cancer cells. Oncogene 22: 3943-3951, 2003.
13. Jin Y, Tian X, Shang Y and Huang P: Inhibition of DLC-1 gene expression by RNA interference in the colon cancer LoVo cell line. Oncol Rep 19: 669-674, 2008.

14. Kim TY, Jackson S, Xiong Y, Whitsett TG, Lobello JR, Weiss GJ, Tran NL, Bang YJ and Der CJ: CRL4A-FBXW5-mediated degradation of DLC1 Rho GTPase-activating protein tumor suppressor promotes non-small cell lung cancer cell growth. Proc Natl Acad Sci USA 110: 16868-16873, 2013

15. Heering J, Erlmann P and Olayioye MA: Simultaneous loss of the DLC1 and PTEN tumor suppressors enhances breast cancer cell migration. Exper Cell Res 315: 2505-2514, 2009.

16. Zhang T, Zheng J, Jiang N, Wang G, Shi Q, Liu C and Lu Y: Overexpression of DLC-1 induces cell apoptosis and proliferation inhibition in the renal cell carcinoma. Cancer Lett 283: 59-67, 2009.

17. Goodison S, Yuan J, Sloan D, Kim R, Li C, Popescu NC and Urquidi V: The RhoGAP protein DLC-1 functions as a metastasis suppressor in breast cancer cells. Cancer Res 65: 6042-6053, 2005.

18. Qin Y, Chu B, Gong W, Wang J, Tang Z, Shen J and Quan Z: Inhibitory effects of deleted in liver cancer 1 gene expression inhibits gallbladder cancer growth through induction of cell cycle arrest and apoptosis. J Gastroenterol Hepatol 29: 964-972, 2013.

19. Chen WT, Yang $\mathrm{CH}$, Wu CC, Huang YC and Chai CY: Aberrant deleted in liver cancer-1 expression is associated with tumor metastasis and poor prognosis in urothelial carcinoma. APMIS 121: 1131-1118, 2013.

20. Tripathi SC, Kaur J, Matta A, Gao X, Sun B, Chauhan SS, Thakar A, Shukla NK, Duggal R, Choudhary AR, et al: Loss of DLC1 is an independent prognostic factor in patients with oral squamous cell carcinoma. Mod Pathol 25: 14-25, 2012.

21. Qian X, Durkin ME, Wang D, Tripathi BK, Olson L, Yang XY, Vass WC, Popescu NC and Lowy DR: Inactivation of the Dlc1 gene cooperates with downregulation of p15INK4b and p16Ink4a, leading to neoplastic transformation and poor prognosis in human cancer. Cancer Res 72: 5900-5911, 2012.

22. Sjoestroem C, Khosravi S, Cheng Y, Safaee Ardekani G, Martinka M and Li G: DLC1 expression is reduced in human cutaneous melanoma and correlates with patient survival. Mod Pathol 27: 1203-1211, 2014.

23. Ren F, Shi H, Zhang G and Zhang R: Expression of deleted in liver cancer 1 and plasminogen activator inhibitor 1 protein in ovarian carcinoma and their clinical significance. J Exp Clin Cancer Res 32: 60, 2013.

24. Wan YW, Sabbagh E, Raese R, Qian Y, Luo D, Denvir J, Vallyathan V, Castranova V and Guo NL: Hybrid models identified a 12-gene signature for lung cancer prognosis and chemoresponse prediction. PloS One 5: e12222, 2010.

25. Shih YP, Liao YC, Lin Y and Lo SH: DLC1 negatively regulates angiogenesis in a paracrine fashion. Cancer Res 70: 8270-8275, 2010 . 